\title{
DIAGNOSTIC CHALLENGES IN CHILD VENTRICULAR TACHYCARDIA
}

\author{
Constantin Iordache, Andreea-Simona Holoc, Claudia-Mihaela Toma, \\ Mihaela Grecu, Alina-Costina Luca \\ ${ }^{1} 1$ st Clinic of Pediatrics, ,, Sf. Maria “ Children's Hospital, \\ „, Gr. T. Popa “, University of Medicine and Pharmacy, Iasi
}

\begin{abstract}
Primary cardiac arrhythmias are much less common in infants and children than in adults, their symptoms can be vague and nonspecific. Although real emergencies because of unstable arrhythmias in children are rare, it is important to identify and properly manage these cases. The study presents the case of a child who was admitted for respiratory symptoms, clinical examination discovering the heart rate of $214 \mathrm{bpm}$. The child became progressively anxious, showing a respiratory effort. The study of electrocardiogram established the diagnosis of anterior fascicular ventricular tachycardia with a tendency to become permanent. The evolution was favorable after the electrical cardioversion and preventive treatment with Verapamil had been performed.
\end{abstract}

Keywords: ventricular tachycardia, child, electrical cardioversion

Ventricular tachycardia is a succession of more than 4 originating distal complexes of Hiss beam bifurcation in the leading specialized tissue or in the ventricular myocardium with a frequency ranging between $120-250 / \mathrm{min}$, having as a mechanism impulse formation disorders or re-entrance phenomena (1).

\section{CASE PRESENTATION}

Patient B.B., aged 6 years and 5 month is admitted for respiratory symptoms: expiratory productive coughing and dyspnea treated at home for 5 days with Klacid and Eurespal. From previous medical history, we discover multiple infections retain the upper airways that did not require hospitalization.

Clinical examination:

- General affected condition $\mathrm{T}=+1.23 \mathrm{DS}$, $\mathrm{G}=+0.87 \mathrm{DS}, \mathrm{PC}=+1,5$;

- Pale skin, Discreet throat congestion;

- Lungs - rough vesicular murmur, rales, bilateral basal, $\mathrm{FR}=32 \mathrm{r} / \mathrm{min}, \mathrm{SaO} 2(-)=99 \%$;
- Heart - rhythmic tachycardial heart sounds, $\mathrm{FC}=214 \mathrm{~b} / \mathrm{min}$;

The remaining devices and systems have indicated normal range. The laboratory data have been within normal range.

Due to increased heart rate, it was decided to perform an electrocardiogram (Fig. 1) which indicated FC: $211 / \mathrm{min}$, regular rhythm, QRS axis of - 90 degrees, the length of QRS complexes is $0.13 \mathrm{sec}$, with regular succession, monomorphic; artrio-ventricular dissociation and the aspect of right bundle branch block associated with left anterior hemiblock.

Stage diagnosis based on the electrocardiogram was difficult to establish. Therefore, the following have been excluded:

- Paroxysmal supraventricular tachycardia anomalously led: although we have the presence of RS (V1-V6) complex, the duration of $\mathrm{RS}$ is over $100 \mathrm{~ms}$ in precordial leads, atrioventricular dissociation in $\mathrm{V} 1$, the initial $\mathrm{R}$ wave in aVR $>40 \mathrm{~ms}$ support the diagnosis of VT. 


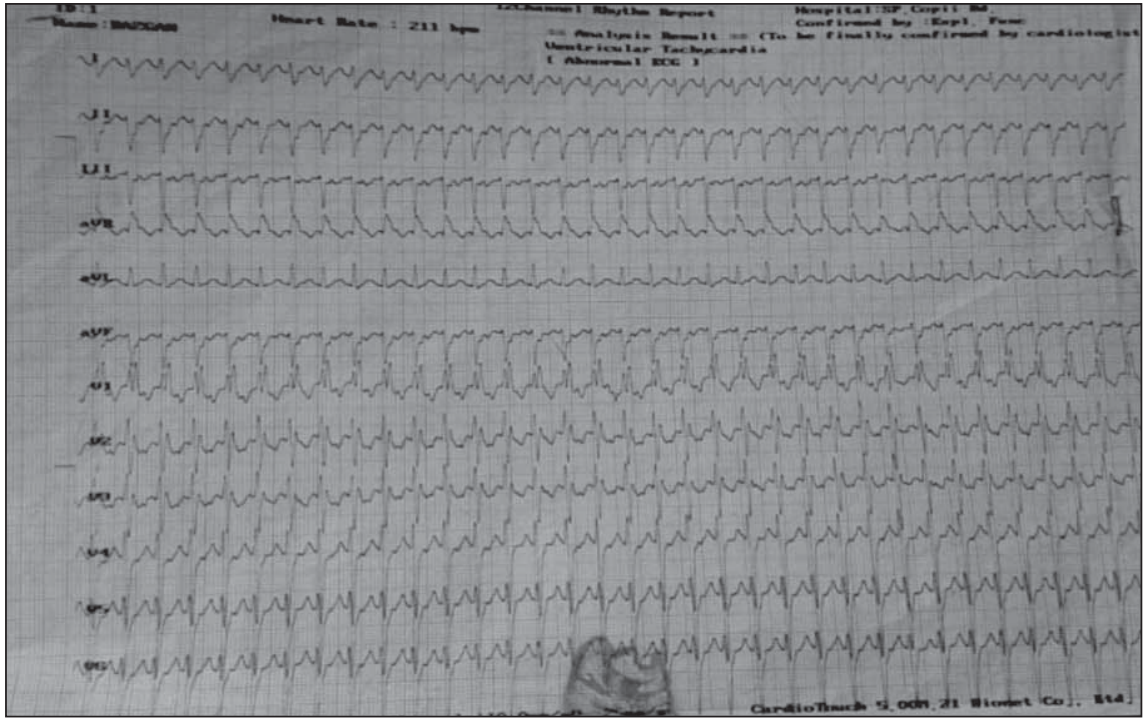

FIGURE 1. Electrocardiogram: anterior fascicular ventricular tachycardia
- Atrial tachycardia with wide QRS complexes Ventricular fibrillation: disorganized ventricular rhythm

- Ventricular flutter: sinusoidal aspect through which ORS seems symmetrical on both sides of the isoelectric line.

- Polymorphic VT

- Accelerated ideoventricular rhythm: it is a monomorphic ventricular tachycardia with frequency in most cases below $120 / \mathrm{min}$, repetitive by accelerated pathological automatism.

Cardio-thoracic radiograph (Fig. 2) showed hiliobasally accentuated pulmonary markings and heart with left arch rectitude (mild cardiomegaly CI: 1.3).

Ecocardiography (Fig. 3) showed a ventricular diastolic dysfunction, tricuspid repression $3^{\text {rd }}$ de-

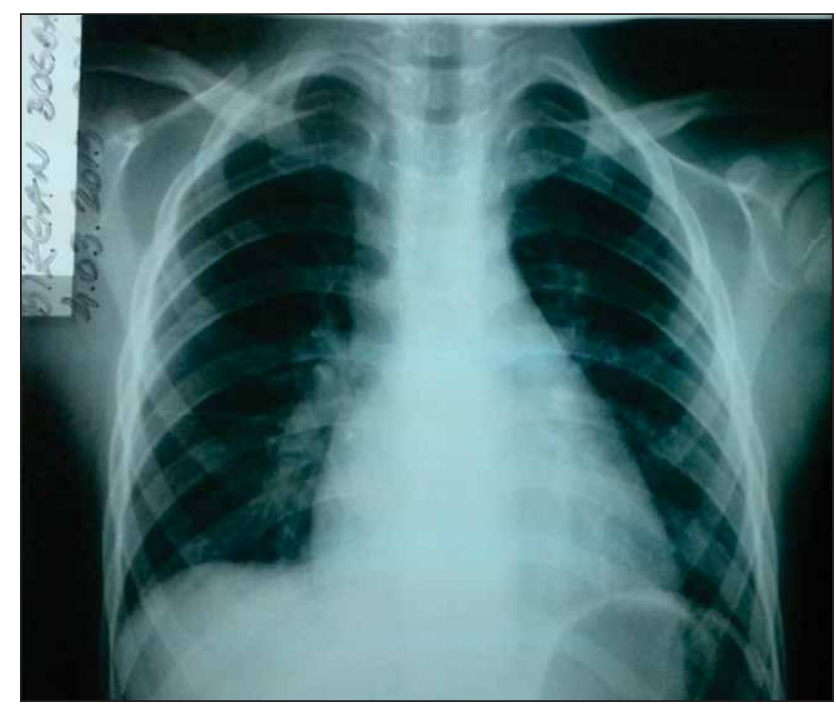

FIGURE 2. Cardio-thoracic radiography gree, patent oval foramen, ventricular septum contraction asynchrony, ejection fraction $60 \%$, shortening fraction of $31 \%$.

Structural changes of the heart do not represent a starting point of ventricular tachycardia but rather a consequence of electrophysiological changes.

Anterior fascicular ventricular tachycardia with atrioventricular dissociation was diagnosed, with a tendency to become permanent, right bundle branch block with left anterior hemiblock. The patient received antibiotic treatment, symptomatics, adenosine $2.5 \mathrm{mg}$ initially, then $5 \mathrm{mg}$, Amiodarone $350 \mathrm{mg}$ x $2 /$ day, tachycardia persists after 24 hours under anti-arrhythmic medication. It was decided that cardioversion should be performed as the functional condition of the child worsens progressively: expiratory dyspnea increased, the extremities being cold and sweaty. General anesthesia with $1 \mathrm{mg}$ iv Midazolamum and Revafil $20 \mathrm{mg}$ intravenously, then $50 \mathrm{~J}$ biphasic external electric shock aisle in sinus rhythm 88/min were performed. (Fig. 4)

We continued daily monitoring of ECG; the patient receiving Verapamil $40 \mathrm{mg} /$ day. In evolution, under medical treatment, the child's general condition remained good, maintaining a heart rate of $100 / \mathrm{min}$.

Ekg at discharge showed a sinus rhythm 100 min, QRS axis: +100 degrees. $P Q=0.14$ sec. Right bundle branch block, negative T waves in DII, DIII, AVF and in left precordial leads (suggesting a slight decrease in cardiac output due to ischemia).

At check-up admissions, patient's condition was good, heart rate of $90 \mathrm{bpm}$, right bundle branch block persisted. 


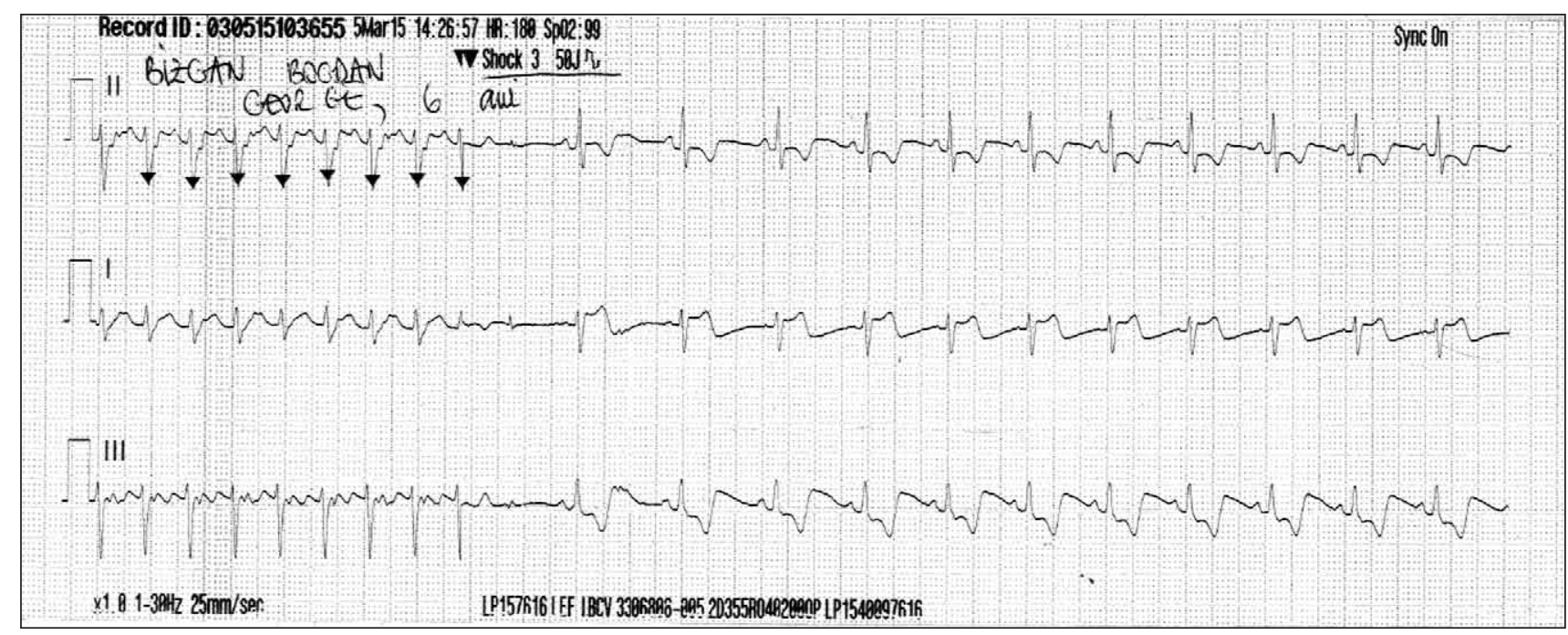

FIGURE 3. EKG during biphasic electrical cardioversion with $50 j$
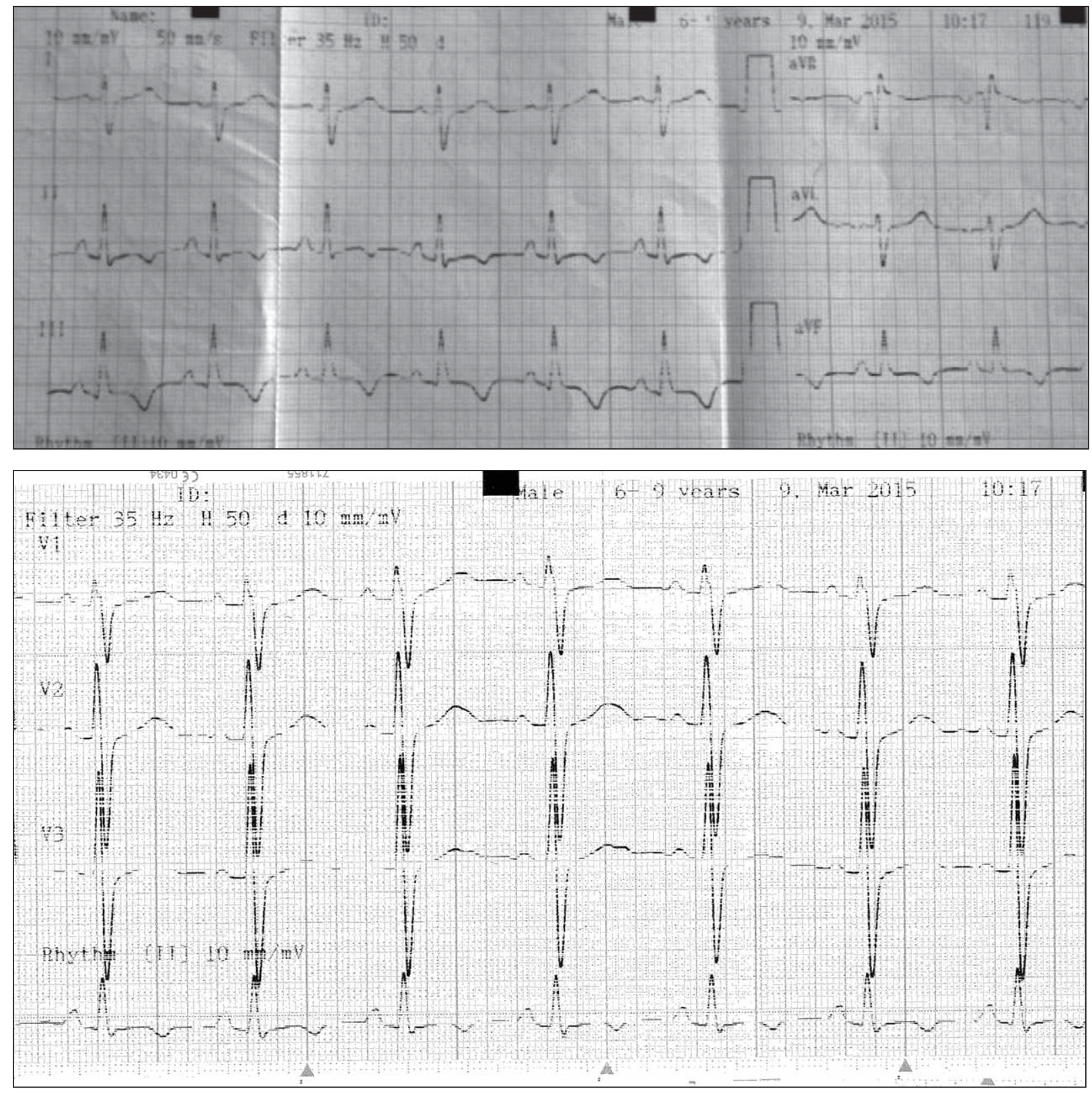

FIGURE 4. EKG at discharge: FC:100/min, BRD 


\section{DISCUSSION}

Ventricular tachycardia is very rare in children, but the impact of this condition on prognosis is extraordinarily high. This is especially important as the higher the heart rate, the more reduced is the ventricular diastole, atrial contribution is missing (atrio-ventricular dissociation), leading to impaired myocardial perfusion and then to sudden death $(2,3)$.

Incessant monomorphic ventricular tachycardia is characterized by the presence of ventricular activity sequences with the same morphology becoming permanent, which is impossible to interrupt under medication (4).

It occurs on a pathological layer (congenital heart disease, ventricular hypertrophy, hypertrophic obstructive cardiomyopathy, arrhythmogenic right ventricular cardiomioparthy, acute myocardial infarction, sarcoidosis, Chagas Disease, Brugada syndrome, long QT syndrome) (11) weakening underventricular fibrillation (5) or in a normal heart being very well tolerated, but it may cause in time dilated cardiomyopathy (6).

By Verekei's algorithm, we can differentiate a ventricular tachycardia from a paroxysmal supraventricular tachycardia by:

- the presence of an initial $\mathrm{R}$ in aVR; where initial $\mathrm{r}$ or q duration is over $40 \mathrm{~ms}$ in aVR; "notched" view of initial slope of the QRS complex predominantely negative in aVR

- ventricular activation-velocity report $(\mathrm{Vi} / \mathrm{Vt})$ $\leq 1$ (slope of first $40 \mathrm{~ms}$ of the QRS complex of aVR/slope of the last $40 \mathrm{~ms}$ of the QRS complex of aVR) (7).

If the patient's condition is stable and is not accompanied by hemodynamic deterioration after
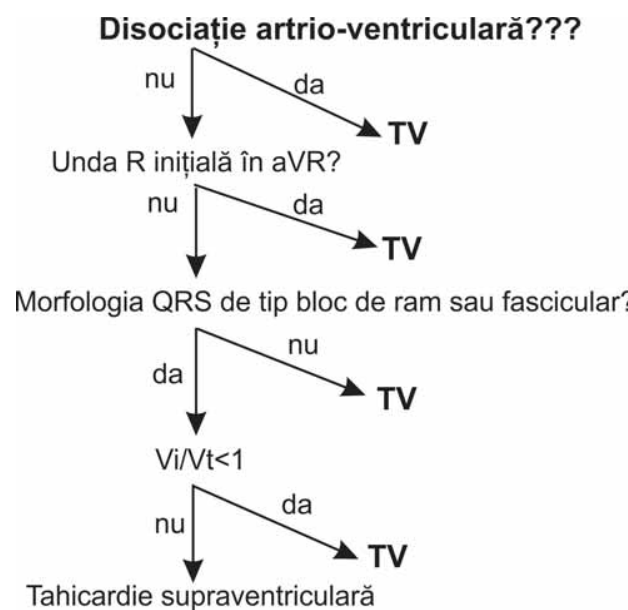

FIGURE 5. The Vereckei algorithm for the diagnosis of ventricular tachycardia proper diagnosis of ventricular tachycardia, cardioversion is attempted with Amiodarone $5 \mathrm{mg} / \mathrm{kg}$ intravenously or Procainamide $15 \mathrm{mg} / \mathrm{kg}$ intravenously (Amiodarone and Procainamide are never administered together) or Lidocaine $1 \mathrm{mg} / \mathrm{kg}$ bolus.

If drug cardioversion was not successful, then the synchronous biphasic electrical cardioversion synchronous is used of $0.5-1 \mathrm{~J} / \mathrm{kg}(2 \mathrm{~J} / \mathrm{kg}$ may be attempted if the initial dose was ineffective); these maneuvers are done only after prior sedation.

Postcardioversion, in fascicular ventricular tachycardia, relapse prevention is achieved by administering orally Verapamil $(9,10)$. If symptoms reappear, or there is a drug-intolerance, invasive treatment methods may be applied:

- Implantable cardio-defibrillator (if there is an increased family risk of sudden death: Long QT syndrome, Brugada syndrome, ventricular tachycardia with severe hemodynamic and ejection fraction $<35 \%$ );

- Radiofrequency ablation treatment (especially indicated in normal cardiac ventricular tachycardia in certain diseases but also in specific pathologies: fascicular tachycardia, idiopathic tachycardia, arrhythmogenic right ventricular dysplasia) (8);

- ablative treatment by other forms of energy: cryoablation, microwaves, laser and ultrasound.

\section{CONCLUSIONS}

Ventricular tachycardia may be an individual pathology on normal heart differently constituted organically or structurally. Good tolerance of symptoms and lack of proper cardiac abnormalities that could have led triggered ventricular tachycardia led to the establishment of anti-arrhythmic medication but were not therapeutically successful. Electrical cardioversion determine the restoring of electrographic path and the normalization of heart rate with a long-term favorable outcome. 


\section{REFERENCES}

1. Silva J.N., Erickson C.C., etc. Management of pediatric tachyarrhythmias on mechanical support. Circ Arrhythm Electrophysiol. 2014 Aug; 7(4):658-63.

2. Noda T., Shimizu W., Taguchi A., et al. Malignant entity of idiopathic ventricular fibrillation and polymorphic ventricular tachycardia initiated by premature extrasystoles originating from the right ventricular outflow tract. J Am Coll Cardiol. 2005; 46(7):1288-94.

3. Saarel E., Gomez C. Fetal arrhythmias. In Macdonald D Clinical electrophysiology in the young. Ed Springer Science and Business Media 2006, 241-257.

4. Shebani S.O., Stafford P., etc. Radiofrequency ablation on veno-arterial extracorporeal life support in treatment of very sick infants with incessant tachymyopathy. Europace. $2015 \mathrm{Apr}$; 17(4):622-7.

5. Chugh S.S., Jui J., Gunson K., Stecker E.C., John B.T., Thompson B. Current burden of sudden cardiac death: multiple source surveillance versus retrospective death certificate-based review in a large U.S. community. J Am Coll Cardiol. 15 2004; 44(6):1268-75.

6. Arya A., Piorkowski C., Sommer P., et al. Idiopathic outflow tract tachycardias: current perspectives. Herz. 2007; 32(3):218-25.
7. Vereckei A., Duray G., Szenasi G., et al. New algorithm using only lead aVR for differential diagnosis of wide QRS complex tachycardia. Heart Rhythm. 2008; 5(1):89-98.

8. Hugh Calkins, Andrew Epstein etc. Catheter ablation of ventricular tachycardia in patients with structural heart disease using cooled radiofrequency energy, J Am Coll Cardiol. 2000; 35(7): 1905-1914.

9. Aiba T., Suyama K., Aihara N., et al. The role of Purkinje and pre-Purkinje potentials in the reentrant circuit of verapamil-sensitive idiopathic LV tachycardia. Pacing Clin Electrophysiol. 2001 Mar; 24(3):333-44.

10. Hiremath G., Foltz R., etc. Verapamil-sensitive idiopathic left ventricular tachycardia in a 6-month-old:unique considerations in diagnosis and management in an infant. Pediatr Emerg Care. 2015 Jan; 31(1):50-3.

11. Luca A. Malformatii congenitale de cord, lordache $C$. Tulburari de ritm si de conducere „in: lordache C. Tratat de pediatrie-editia I, Editura „Gr.T. Popa”, Iaşi 2011: 322-370, 395-424. 